\title{
Optimal Investment and Consumption Decisions under the Constant Elasticity of Variance Model
}

\author{
Hao Chang, ${ }^{1}$ Xi-min Rong, ${ }^{2}$ Hui Zhao, ${ }^{2}$ and Chu-bing Zhang $^{3}$ \\ ${ }^{1}$ Department of Mathematics, Tianjin Polytechnic University, Tianjin 300387, China \\ ${ }^{2}$ School of Science, Tianjin University, Tianjin 300072, China \\ ${ }^{3}$ School of Business, Tianjin University of Finance and Economics, Tianjin 30022, China \\ Correspondence should be addressed to Hao Chang; ch8683897@126.com
}

Received 21 July 2013; Accepted 3 October 2013

Academic Editor: Fazal M. Mahomed

Copyright (C) 2013 Hao Chang et al. This is an open access article distributed under the Creative Commons Attribution License, which permits unrestricted use, distribution, and reproduction in any medium, provided the original work is properly cited.

\begin{abstract}
We consider an investment and consumption problem under the constant elasticity of variance (CEV) model, which is an extension of the original Merton's problem. In the proposed model, stock price dynamics is assumed to follow a CEV model and our goal is to maximize the expected discounted utility of consumption and terminal wealth. Firstly, we apply dynamic programming principle to obtain the Hamilton-Jacobi-Bellman (HJB) equation for the value function. Secondly, we choose power utility and logarithm utility for our analysis and apply variable change technique to obtain the closed-form solutions to the optimal investment and consumption strategies. Finally, we provide a numerical example to illustrate the effect of market parameters on the optimal investment and consumption strategies.
\end{abstract}

\section{Introduction}

In the classical Merton's portfolio optimization problems [1, $2]$, an investor dynamically allocates his wealth between one risk asset and one risk-free asset and chooses an optimal consumption rate to maximize total expected discounted utility of consumption. But in the Merton's model, there are no transaction costs and borrowing constraints and noshorting constraints. Since the pioneer work of Merton, the investment and consumption problems have inspired literally hundreds of extensions and applications. For example, introducing transaction costs into the investment and consumption problems, one can refer to Shreve and Soner [3], Akian et al. [4], and Janeček and Shreve [5]. Some authors as well investigated the optimal consumption problem with borrowing constraints; see Fleming and Zariphopoulou [6], Vila and Zariphopoulou [7], and Yao and Zhang [8]. However, the above mentioned models generally were studied under the assumption that risky asset price dynamics was driven by a geometric Brownian motion (GBM).

The constant elasticity of variance (CEV) model is a natural extension of the GBM. Compared with the GBM, the advantages of the CEV model are that the volatility rate has correlation with the risky asset price and can explain volatility smile. The CEV model was originally proposed by Cox and Ross [9] as an alternative diffusion process for European option pricing. The CEV model was usually applied to analyze the option pricing formula, see; for example, Schroder [10], Lo et al. [11], Phelim and Yisong [12], and Davydov and Linetsky [13]. In the recent years, the CEV model has been introduced into annuity contracts and the optimal investment strategies in the utility framework are investigated by applying dynamic programming principle. For more detailed discussion, one can refer to Xiao et al. [14], Gao [15, 16], Gu et al. [17], Lin and Li [18], Gu et al. [19], Jung and Kim [20] and Zhao and Rong [21]. However, the application of the CEV model to an investment and consumption problem has not been reported in the existing academic articles.

In this paper, we introduce a CEV model into an investment and consumption problem and optimally allocate the wealth between one risk-free asset and one risky asset, whose price process is supposed to follow a CEV model. Our goal is to maximize the expected discounted utility of consumption 
and terminal wealth. Dynamic programming principle is applied to obtain the HJB equation for the value function. Owing to the introducing of consumption factor and the CEV model, the HJB equation derived is much more difficult to deal with than the one obtained by Gao [15]. Inspired by the techniques of Gao [15] and Liu [22], we transform the nonlinear second-order partial differential equation into a linear one, which is easy to tackle. In the techniques of tackling the CEV model, one of the most important innovations in this paper is to suppose that the structure of the solution to (22) is of the expression form (23) and prove that (22) and (25) are equivalent. Secondly, we derive the closedform solutions to the optimal investment and consumption strategies for power utility and logarithm utility by applying variable change technique. Finally, we propose a numerical example to illustrate the properties and sensitivities of the optimal investment and consumption strategy on market parameters. There are three main contributions in this paper: (i) we first consider an investment and consumption problem under a CEV process and obtain the closed-form solutions of the optimal investment and consumption strategies in the power and logarithm utility cases; (ii) we use the same approach as Liu [22] to solve (22), which is very difficult to solve directly; (iii) we provide a numerical example to illustrate our results.

The rest of this paper is organized as follows. In Section 2, we formulate the financial market and propose the optimization problem. In Section 3, we apply dynamic programming principle to obtain the HJB equation and investigate the optimal investment and consumption strategies in the power and logarithm utility cases. Section 4 provides a numerical example and Section 5 concludes the paper.

\section{Problem Formulation}

In this section, we propose the problem formulation of optimal investments and consumption decisions with a CEV process.

We consider a financial market where two assets are traded continuously over $[0, T]$. One asset is a bond with price $P_{t}$ at time $t$, whose price process $P_{t}$ satisfies

$$
d P_{t}=r P_{t} d t, \quad P_{0}^{0}=p_{0}>0,
$$

where the constant $r>0$ is the interest rate of the bond.

The other asset is a stock with prices $S_{t}$ at time $t$, whose price process $S_{t}$ is given by the following constant elasticity of variance model $(\mathrm{CEV})$ :

$$
d S_{t}=S_{t}\left(\mu d t+k S_{t}^{\gamma} d W_{t}\right), \quad S_{0}=s_{0}>0,
$$

where $\mu(\mu>r)$ is an expected instantaneous return rate of the stock. $k$ and $\gamma$ are constant parameters and the elasticity parameter $\gamma$ satisfies the general condition: $\gamma \leqslant 0 . k S_{t}^{\gamma}$ is defined as the instantaneous volatility of the stock. $W_{t}$ is a one-dimensional standard adapted Brownian motion defined on a filtered complete probability space $\left(\Omega, \mathscr{F}, P,\left\{\mathscr{F}_{t}\right\}_{0 \leqslant t \leqslant T}\right)$, where $\left\{\mathscr{F}_{t}\right\}_{0 \leqslant t \leqslant T}$ is a $\sigma$-algebra generated by Brownian motion $W_{t}$.
Remark 1. Noting that there are four special interpretations for the elasticity parameter $\gamma$ : (i) if $\gamma=0$, the CEV model is reduced to a geometric Brownian motion (GBM); (ii) if $\gamma=$ -1 , it is the Ornstein-Uhlenbeck process; (iii) if $\gamma=-1 / 2$, it is the model first presented by Cox and Ross [9] as an alternative diffusion process for valuation of options; (iv) if $\gamma<0$, this means that the instantaneous volatility $k S_{t}^{\gamma}$ increases as the stock price decreases and can generate a distribution with a fatter left tail.

Assume that the investor invests the market value of his wealth $\pi_{t}$ into the stock at time $t, t \in[0, T]$. Clearly, the amount invested in the bond is $X_{t}-\pi_{t}$, in which $X_{t}$ represents the wealth of the investor at time $t$. Suppose that short-selling of stocks and borrowing at the interest rate of the bond are allowed and transaction cost is not taken into consideration. We also introduce the consumption rate denoted by $C_{t}$. The wealth process $X_{t}$ corresponding to trading strategy $\left(\pi_{t}, C_{t}\right)$ is subject to the following equation:

$$
d X_{t}=\left(X_{t}-\pi_{t}\right) \frac{d P_{t}}{P_{t}}+\pi_{t} \frac{d S_{t}}{S_{t}}-C_{t} d t
$$

namely, we have

$$
\begin{array}{r}
d X_{t}=\left[r X_{t}+(\mu-r) \pi_{t}-C_{t}\right] d t+\pi_{t} k S_{t}^{\gamma} d W_{t}, \\
X_{0}=x_{0}>0 .
\end{array}
$$

Definition 2 (admissible strategy). An investment and consumption $\left(\pi_{t}, C_{t}\right)$ is admissible if the following conditions are satisfied:

(i) $\left(\pi_{t}, C_{t}\right)$ is $\mathscr{F}_{t}$-progressively measurable and $\int_{0}^{T} \pi_{t}^{2} d t<\infty, \int_{0}^{T} C_{t} d t<\infty$, a.s. $\forall T>0 ;$

(ii) $E\left[\int_{0}^{T}\left(\pi_{t} k S_{t}^{\gamma}\right)^{2} d t\right]<\infty$;

(iii) For $\forall\left(\pi_{t}, C_{t}\right)$, stochastic differential equation (4) has a unique solution.

Assume that the set of all admissible investment and consumption strategies $\left(\pi_{t}, C_{t}\right)$ is denoted by $\Gamma=\left\{\left(\pi_{t}, C_{t}\right): 0 \leqslant\right.$ $t \leqslant T\}$. Mathematically, an investor wishes to maximize the following objective function:

$$
\underset{\left\{\pi_{t}, C_{t}\right\} \in \Gamma}{\operatorname{Maximize}} \mathbb{E}\left[\alpha \int_{0}^{T} e^{-\beta t} U_{1}\left(C_{t}\right) d t+(1-\alpha) e^{-\beta T} U_{2}\left(X_{T}\right)\right],
$$

where utility function $U(\cdot)$ is assumed to be strictly concave and continuously differentiable on $(-\infty,+\infty)$ and $\beta$ is the subjective discount. The parameter $\alpha$ determines the relative importance of the intermediate consumption and the terminal wealth. Since $U(\cdot)$ is strictly concave, there exists a unique optimal trading strategy $\left(\pi_{t}, C_{t}\right)$ which maximizes (5).

In this paper, we consider two-type utility functions $U(\cdot)$. One is power utility function defined by $U(x)=x^{\eta} / \eta, \eta<$ 1 and $\eta \neq 0$. The other is logarithm utility function given by $U(x)=\ln x$. 


\section{The Closed-Form Solution}

In this section, we apply dynamic programming principle to derive the HJB equation for the value function and investigate the optimal investment and consumption policies for problem (5) in the power and logarithm utility cases.

The value function is defined as

$$
\begin{aligned}
& H(t, s, x) \\
& =\sup _{\left\{\pi_{t}, C_{t}\right\} \in \Gamma} \mathbb{E}\left\{\alpha \int_{0}^{T} e^{-\beta t} U_{1}\left(C_{t}\right) d t\right. \\
& \left.\quad+(1-\alpha) e^{-\beta T} U_{2}\left(X_{T}\right) \mid S_{t}=s, X_{t}=x\right\},
\end{aligned}
$$

with boundary condition given by $H(T, s, x)=(1-$ $\alpha) e^{-\beta T} U_{2}(x)$.

Using dynamic programming principle, one can get the HJB equation as follows:

$$
\begin{aligned}
\sup _{\left\{\pi_{t}, C_{t}\right\} \in \Gamma}\left\{\frac{\partial H}{\partial t}\right. & +\left[(\mu-r) \pi_{t}+r x-C_{t}\right] \frac{\partial H}{\partial x} \\
& +\frac{1}{2}\left(\pi_{t} k s^{\gamma}\right)^{2} \frac{\partial^{2} H}{\partial x^{2}}+\mu s \frac{\partial H}{\partial s}+\frac{1}{2}\left(k s^{\gamma+1}\right)^{2} \frac{\partial^{2} H}{\partial s^{2}} \\
& \left.+\pi_{t} k^{2} s^{2 \gamma+1} \frac{\partial^{2} H}{\partial x \partial s}+\alpha e^{-\beta t} U_{1}\left(C_{t}\right)\right\}=0,
\end{aligned}
$$

where $\partial H / \partial t, \partial H / \partial x, \partial^{2} H / \partial x^{2}, \partial H / \partial s, \partial^{2} H / \partial s^{2}$, and $\partial^{2} H / \partial x \partial s$ denote first-order and second-order partial derivatives with respect to the variables $t, s$, and $x$, respectively.

The first-order maximizing conditions for the optimal value is given by

$$
\begin{gathered}
\pi_{t}^{*}=-\frac{\mu-r}{\left(k s^{\gamma}\right)^{2}} \cdot \frac{\partial H / \partial x}{\partial^{2} H / \partial x^{2}}-s \cdot \frac{\partial^{2} H / \partial x \partial s}{\partial^{2} H / \partial x^{2}}, \\
U^{\prime}\left(C_{t}^{*}\right)=\frac{\partial H / \partial x}{\alpha e^{-\beta t}} .
\end{gathered}
$$

Introducing (8) into (7), we obtain

$$
\begin{aligned}
\frac{\partial H}{\partial t} & +r x \frac{\partial H}{\partial x}+\mu s \frac{\partial H}{\partial s}+\frac{1}{2}\left(k s^{\gamma+1}\right)^{2} \frac{\partial^{2} H}{\partial s^{2}} \\
& -\frac{1}{2} \cdot \frac{\left[(\mu-r) \partial H / \partial x+k^{2} s^{2 \gamma+1} \partial^{2} H / \partial x \partial s\right]^{2}}{k^{2} s^{2 \gamma} \partial^{2} H / \partial x^{2}} \\
& -C_{t}^{*} \frac{\partial H}{\partial x}+\alpha e^{-\beta t} U_{1}\left(C_{t}^{*}\right)=0 .
\end{aligned}
$$

Here, we notice that the stochastic control problem has been transformed into a nonlinear second-order partial differential equation; yet it is difficult to solve it. In the following subsection, we choose power utility and logarithm utility for our analysis, respectively, and try to obtain the closed-form solutions to (9).
3.1. Power Utility. Power utility function is defined as

$$
U_{1}(x)=U_{2}(x)=\frac{x^{\eta}}{\eta}, \quad \eta<1, \quad \eta \neq 0 .
$$

For (9), we can conjecture a solution with the following structure:

$$
H(t, s, x)=e^{-\beta t} \frac{x^{\eta}}{\eta} f(t, s), \quad f(T, s)=1-\alpha .
$$

Then

$$
\begin{aligned}
& \frac{\partial H}{\partial t}=-\beta e^{-\beta t} \frac{x^{\eta}}{\eta} f(t, s)+e^{-\beta t} \frac{x^{\eta}}{\eta} \cdot \frac{\partial f}{\partial t}, \\
& \frac{\partial H}{\partial x}=e^{-\beta t} x^{\eta-1} f, \quad \frac{\partial^{2} H}{\partial x^{2}}=(\eta-1) e^{-\beta t} x^{\eta-2} f \\
& \frac{\partial H}{\partial s}=e^{-\beta t} \frac{x^{\eta}}{\eta} \cdot \frac{\partial f}{\partial s}, \quad \frac{\partial^{2} H}{\partial s^{2}}=e^{-\beta t} \frac{x^{\eta}}{\eta} \cdot \frac{\partial^{2} f}{\partial s^{2}} \\
& \frac{\partial^{2} H}{\partial s \partial x}=e^{-\beta t} x^{\eta-1} \frac{\partial f}{\partial s} .
\end{aligned}
$$

Therefore, (8) is rewritten as

$$
\begin{gathered}
\pi_{t}^{*}=\frac{1}{1-\eta} \cdot \frac{\mu-r}{\left(k s^{\gamma}\right)^{2}} x+\frac{s}{1-\eta} \cdot \frac{\partial f / \partial s}{f} x, \\
C_{t}^{*}=\alpha^{1 /(1-\eta)} f^{-1 /(1-\eta)} x .
\end{gathered}
$$

Putting these partial derivatives and the optimal policy (13) into (9), we get

$$
\begin{aligned}
e-\beta t \frac{x^{\eta}}{\eta}\left[\frac{\partial f}{\partial t}\right. & +(\eta r-\beta) f+\mu s \frac{\partial f}{\partial s}+\frac{1}{2} k^{2} s^{2 \gamma+2} \frac{\partial^{2} f}{\partial s^{2}} \\
& -\frac{\eta}{2(\eta-1)}\left(\frac{\mu-r}{k s^{\gamma}}\right)^{2} f-\frac{\eta}{\eta-1}(\mu-r) s \frac{\partial f}{\partial s} \\
& -\frac{\eta}{2(\eta-1)} k^{2} s^{2 \gamma+2} \frac{\partial f / \partial s}{f} \\
& \left.+(1-\eta) \alpha^{1 /(1-\eta)} f^{\eta /(\eta-1)}\right]=0 .
\end{aligned}
$$

Eliminating the dependence on $x$, we obtain

$$
\begin{aligned}
f_{t} & +(\eta r-\beta) f+\mu s f_{s}+\frac{1}{2} k^{2} s^{2 \gamma+2} f_{s s} \\
& -\frac{\eta}{2(\eta-1)}\left(\frac{\mu-r}{k s^{\gamma}}\right)^{2} f-\frac{\eta}{\eta-1}(\mu-r) s f_{s} \\
& -\frac{\eta}{2(\eta-1)} k^{2} s^{2 \gamma+2} \frac{\partial f / \partial s}{f}+(1-\eta) \alpha^{1 /(1-\eta)} f^{\eta /(\eta-1)}=0 .
\end{aligned}
$$

Inspired by the approach of Gao [15], we can use the following power transform and variable change technique. So letting

$$
f(t, s)=g(t, y), \quad y=s^{-2 \gamma},
$$


we get

$$
\begin{gathered}
\frac{\partial f}{\partial t}=\frac{\partial g}{\partial t}, \quad \frac{\partial f}{\partial s}=\frac{\partial g}{\partial y}(-2 \gamma) s^{-2 \gamma-1} \\
\frac{\partial^{2} f}{\partial s^{2}}=\frac{\partial^{2} g}{\partial y^{2}} 4 \gamma^{2} s^{-4 \gamma-2}+\frac{\partial g}{\partial y}(-2 \gamma)(-2 \gamma-1) s^{-2 \gamma-2}
\end{gathered}
$$

Introducing these derivatives into (15), we obtain

$$
\begin{aligned}
\frac{\partial g}{\partial t}+ & (\eta r-\beta) g-\frac{\eta}{2(\eta-1)}\left(\frac{\mu-r}{k}\right)^{2} y g \\
& +\left(\frac{\eta}{\eta-1} 2 \gamma(\mu-r)-2 \gamma \mu\right) y \frac{\partial g}{\partial y}+\gamma(2 \gamma+1) k^{2} \frac{\partial g}{\partial y} \\
& +2 \gamma^{2} k^{2} y \frac{\partial^{2} g}{\partial y^{2}}-\frac{2 \eta}{\eta-1} \gamma^{2} k^{2} y \frac{(\partial g / \partial y)^{2}}{g} \\
& +(1-\eta) \alpha^{-1 /(\eta-1)} g^{\eta /(\eta-1)}=0, \quad g(T, y)=1-\alpha .
\end{aligned}
$$

In addition, we use the following variable change technique. Assume that

$$
g(t, y)=h(t, y)^{1-\eta}, \quad h(T, y)=(1-\alpha)^{1 /(1-\eta)} \text {. }
$$

Then

$$
\begin{gathered}
\frac{\partial g}{\partial t}=(1-\eta) h^{-\eta} \frac{\partial h}{\partial t}, \quad \frac{\partial g}{\partial y}=(1-\eta) h^{-\eta} \frac{\partial h}{\partial y}, \\
\frac{\partial^{2} g}{\partial y^{2}}=(1-\eta)(-\eta) h^{-\eta-1}\left(\frac{\partial h}{\partial y}\right)^{2}+(1-\eta) h^{-\eta} \frac{\partial^{2} h}{\partial y^{2}}
\end{gathered}
$$

Substituting these derivatives back into (18), we have

$$
\begin{aligned}
(1-\eta) h^{-\eta}\left[\frac{\partial h}{\partial t}+\left(\frac{\eta r-\beta}{1-\eta}+\frac{\eta}{2(\eta-1)^{2}}\left(\frac{\mu-r}{k}\right)^{2} y\right) h\right. \\
+\left(\frac{\eta}{\eta-1} 2 \gamma(\mu-r)-2 \gamma \mu\right) y \frac{\partial h}{\partial y} \\
+\gamma(2 \gamma+1) k^{2} \frac{\partial h}{\partial y}+2 \gamma^{2} k^{2} y \frac{\partial^{2} h}{\partial y^{2}} \\
\left.+\alpha^{1 /(1-\eta)}\right]=0 .
\end{aligned}
$$

So we obtain the following partial differential equation:

$$
\begin{gathered}
\frac{\partial h}{\partial t}+\left(\frac{\eta r-\beta}{1-\eta}+\frac{\eta}{2(\eta-1)^{2}}\left(\frac{\mu-r}{k}\right)^{2} y\right) h \\
+\left(\frac{\eta}{\eta-1} 2 \gamma(\mu-r)-2 \gamma \mu\right) y \frac{\partial h}{\partial y}+\gamma(2 \gamma+1) k^{2} \frac{\partial h}{\partial y} \\
+2 \gamma^{2} k^{2} y \frac{\partial^{2} h}{\partial y^{2}}+\alpha^{1 /(1-\eta)}=0, \\
h(T, y)=(1-\alpha)^{1 /(1-\eta)} .
\end{gathered}
$$

Noting that (22) has been a linear second-order partial differential equation, it is still very difficult to solve it directly. Inspired by the approach proposed by Liu [22], we try to fit a solution to (22) and we have the following Lemma.

Lemma 3. Assume that $h(t, y)=\alpha^{1 /(1-\eta)} \int_{t}^{T} \tilde{h}(u, y) d u+(1-$ $\alpha)^{1 /(1-\eta)} \widetilde{h}(t, y)$ is a solution of (22); then one can prove that $\tilde{h}(t, y)$ satisfies the equation:

$$
\begin{aligned}
\frac{\partial \tilde{h}}{\partial t} & +\left(\frac{\eta r-\beta}{1-\eta}+\frac{\eta}{2(\eta-1)^{2}}\left(\frac{\mu-r}{k}\right)^{2} y\right) \widetilde{h} \\
& +\left(\frac{\eta}{\eta-1} 2 \gamma(\mu-r)-2 \gamma \mu\right) y \frac{\partial \tilde{h}}{\partial y} \\
& +\gamma(2 \gamma+1) k^{2} \frac{\partial \tilde{h}}{\partial y}+2 \gamma^{2} k^{2} y \frac{\partial^{2} \widetilde{h}}{\partial y^{2}}=0, \quad \tilde{h}(T, y)=1 .
\end{aligned}
$$

Proof. Define differential operator $\nabla$ on any function $h(t, y)$ by

$\nabla h(t, y)$

$$
\begin{aligned}
= & 2 \gamma^{2} k^{2} y \frac{\partial^{2} h}{\partial y^{2}}+\left(\frac{\eta}{\eta-1} 2 \gamma(\mu-r)-2 \gamma \mu\right) y \frac{\partial h}{\partial y} \\
& +\gamma(2 \gamma+1) k^{2} \frac{\partial h}{\partial y} \\
& +\left(\frac{\eta r-\beta}{1-\eta}+\frac{\eta}{2(\eta-1)^{2}}\left(\frac{\mu-r}{k}\right)^{2} y\right) h .
\end{aligned}
$$

Then (22) can be rewritten as

$$
\begin{gathered}
\frac{\partial h(t, y)}{\partial t}+\nabla h(t, y)+\alpha^{1 /(1-\eta)}=0 \\
h(T, y)=(1-\alpha)^{1 /(1-\eta)} .
\end{gathered}
$$


On the other hand, we find that

$$
\begin{aligned}
\frac{\partial h(t, y)}{\partial t}= & -\alpha^{1 /(1-\eta)} \tilde{h}(t, y)+(1-\alpha)^{1 /(1-\eta)} \frac{\partial \widetilde{h}(t, y)}{\partial t} \\
= & \alpha^{1 /(1-\eta)}\left[\int_{t}^{T} \frac{\partial \widetilde{h}(u, y)}{\partial u} d u-\widetilde{h}(T, y)\right] \\
& +(1-\alpha)^{1 /(1-\eta)} \frac{\partial \widetilde{h}(t, y)}{\partial t} \\
\nabla h(t, y)= & \alpha^{1 /(1-\eta)} \int_{t}^{T} \nabla \tilde{h}(u, y) d u \\
& +(1-\alpha)^{1 /(1-\eta)} \cdot \nabla \widetilde{h}(t, y) .
\end{aligned}
$$

Further, (25) is reduced to

$$
\begin{gathered}
\alpha^{1 /(1-\eta)}\left[\int_{t}^{T}\left(\frac{\partial \widetilde{h}(u, y)}{\partial u}+\nabla \tilde{h}(u, y)\right) d u-\widetilde{h}(T, y)+1\right] \\
+(1-\alpha)^{1 /(1-\eta)}\left[\frac{\partial \widetilde{h}(t, y)}{\partial t}+\nabla \widetilde{h}(t, y)\right]=0 .
\end{gathered}
$$

Then we obtain

$$
\frac{\partial \widetilde{h}(t, y)}{\partial t}+\nabla \widetilde{h}(t, y)=0, \quad \widetilde{h}(T, y)=1
$$

Therefore, (23) holds.

Lemma 4. Suppose that a solution to (23) is of the form $\widetilde{h}(t, y)=e^{A(t)+B(t) y}$, with terminal conditions $A(T)=0$ and $B(T)=0$; then $A(t)$ and $B(t)$ are given by (31) and (32), respectively.

Proof. Putting $\widetilde{h}(t, y)=e^{A(t)+B(t) y}$ into (23) yields

$$
\begin{aligned}
\tilde{h}(t, y)\left[y \left(\frac{d B(t)}{d t}+2 \gamma^{2} k^{2} B^{2}(t)\right.\right. \\
+\left(\frac{\eta}{\eta-1} 2 \gamma(\mu-r)-2 \gamma \mu\right) B(t) \\
\left.+\frac{1}{2} \frac{\eta}{(\eta-1)^{2}}\left(\frac{\mu-r}{k}\right)^{2}\right) \\
\left.+\frac{d A(t)}{d t}+\frac{\eta r-\beta}{1-\eta}+\gamma(2 \gamma+1) k^{2} B(t)\right]=0
\end{aligned}
$$

We can decompose (29) into two equations in order to eliminate the dependence on $y$ :

$$
\begin{aligned}
& \frac{d B(t)}{d t}+2 \gamma^{2} k^{2} B^{2}(t)+\left(\frac{\eta}{\eta-1} 2 \gamma(\mu-r)-2 \gamma \mu\right) B(t) \\
& +\frac{1}{2} \cdot \frac{\eta}{(\eta-1)^{2}}\left(\frac{\mu-r}{k}\right)^{2}=0, \quad B(T)=0 ; \\
& \frac{d A(t)}{d t}+\frac{\eta r-\beta}{1-\eta}+\gamma(2 \gamma+1) k^{2} B(t)=0, \quad A(T)=0 .
\end{aligned}
$$

Solving the above two equations by using the same approach as Gao [15], we obtain

$$
\begin{gathered}
A(t)=\left\{\lambda_{1} \gamma(2 \gamma+1) k^{2}+\frac{\eta r-\beta}{1-\eta}\right\}(T-t) \\
+\ln \left\{\frac{\lambda_{2}-\lambda_{1}}{\lambda_{2}-\lambda_{1} e^{-2 \gamma^{2} k^{2}\left(\lambda_{1}-\lambda_{2}\right)(T-t)}}\right\}^{(2 \gamma+1) / 2 \gamma}, \\
B(t)=k^{-2} I(t),
\end{gathered}
$$

where

$$
\begin{aligned}
I(t) & =\frac{\lambda_{1} \lambda_{2}\left(1-e^{-2 \gamma^{2} k^{2}\left(\lambda_{1}-\lambda_{2}\right)(T-t)}\right)}{\lambda_{2}-\lambda_{1} e^{-2 \gamma^{2} k^{2}\left(\lambda_{1}-\lambda_{2}\right)(T-t)}}, \\
\lambda_{1,2} & =\frac{(\mu-r \eta) \pm \sqrt{(1-\eta)\left(\mu^{2}-r^{2} \eta\right)}}{2 \gamma k^{2}(1-\eta)} .
\end{aligned}
$$

As a result, Lemma 4 is completed.

Taking $f(t, s)=g(t, y)=h(t, y)^{1-\eta}$ and their relationships into consideration, we derive

$$
\begin{aligned}
\frac{\partial H / \partial x}{\partial^{2} H / \partial x^{2}} & =-\frac{1}{1-\eta} x, \\
\frac{\partial^{2} H / \partial x \partial s}{\partial^{2} H / \partial x^{2}} & =\frac{x}{\eta-1} \cdot \frac{\partial f / \partial s}{f}=\frac{x}{\eta-1} \cdot(-2 \gamma) s^{-2 \gamma-1} \frac{\partial g / \partial y}{g} \\
& =2 \gamma s^{-2 \gamma-1} \frac{\partial h / \partial y}{h} x .
\end{aligned}
$$

Therefore, the optimal investment strategy is

$$
\pi_{t}^{*}=\frac{1}{1-\eta} \cdot \frac{\mu-r}{\left(k S_{t}^{\gamma}\right)^{2}} X_{t}-2 \gamma S_{t}^{-2 \gamma} \frac{\partial h / \partial y}{h} X_{t} .
$$

So, we can summarize the above results in the following theorem.

Theorem 5. If utility function is given by $U_{1}(x)=U_{2}(x)=$ $x^{\eta} / \eta, \eta<1$ and $\eta \neq 0$, the optimal investment and consumption strategy of the problem (5) is

$$
\begin{aligned}
& \pi_{t}^{*}=\frac{1}{1-\eta} \cdot \frac{\mu-r}{\left(k S_{t}^{\gamma}\right)^{2}} X_{t}\left(1-\frac{2 \gamma(1-\eta) k^{2}}{\mu-r} \cdot \frac{\partial h / \partial y}{h}\right), \\
& C_{t}^{*}=\alpha^{1 /(1-\eta)} h^{-1} X_{t},
\end{aligned}
$$


where $h=h(t, y)=\alpha^{1 /(1-\eta)} \int_{t}^{T} e^{A(t)+B(t) y} d t+(1-$ $\alpha)^{1 /(1-\eta)} e^{A(t)+B(t) y}, y=S_{t}^{-2 \gamma}$, and $A(t)$ and $B(t)$ are determined by (31) and (32), respectively.

Remark 6. Noting that a CEV model includes several existing stochastic processes as special cases, for example, geometric Brownian motion (GBM), Ornstein-Uhlenbeck process, and an alternative diffusion process investigated by Cox and Ross [9], the closed-form expression (36) obtained is the general framework of the optimal investment and consumption strategies when stock price dynamics is given by the above stochastic processes.

Remark 7. It can be seen from (36) that the optimal investment strategy $\pi_{t}^{*}$ can be decomposed into two terms. One term is $(1 /(1-\eta)) \cdot\left((\mu-r) /\left(k S_{t}^{\gamma}\right)^{2}\right) X_{t}$, which has an analogical form of the optimal policy under a GBM model. The other term is $1-\left(\left(2 \gamma(1-\eta) k^{2}\right) /(\mu-r)\right) \cdot((\partial h / \partial y) / h)$, which can be called as modification factor denoted by $\widetilde{M}(t)$. In addition, we notice that two terms are influenced by stock price $S_{t}$.

In order to compare our results with those in the existing literature, we discuss several special cases for Theorem 5 .

Special Case 1. If $\gamma \rightarrow 0$, the CEV model is reduced to a GBM. In addition, we obtain $y=1$ and

$$
\begin{aligned}
& B(t)=\frac{\eta}{2(\eta-1)^{2}}\left(\frac{\mu-r}{k}\right)^{2}(T-t), \\
& A(t)=\frac{\eta r-\beta}{1-\eta}(T-t)
\end{aligned}
$$

Letting

$$
\theta=\frac{\eta}{2(\eta-1)^{2}}\left(\frac{\mu-r}{k}\right)^{2}+\frac{\eta r-\beta}{1-\eta}
$$

then we have

$$
\begin{array}{r}
h(t, y)=h(t)=\alpha^{1 /(1-\eta)} \cdot \frac{1}{\theta}\left(e^{\theta(T-t)}-1\right) \\
+(1-\alpha)^{1 /(1-\eta)} e^{\theta(T-t)},
\end{array}
$$

and $(\partial h / \partial y) / h=0$. Therefore, $(36)$ is reduced to

$$
\pi_{t}^{*}=\frac{1}{1-\eta} \cdot \frac{\mu-r}{k^{2}} X_{t}, \quad C_{t}^{*}=\alpha^{1 /(1-\eta)} h^{-1}(t) X_{t},
$$

where $h(t)$ is given by (39).

Special Case 2. If $\alpha \rightarrow 0$, then $h(t, y)=e^{A(t)+B(t) y}$ and $(\partial h / \partial y) / h=B(\mathrm{t})$. As a result, the optimal consumption policy is $C_{t}^{*}=0$ and the optimal investment strategy is given by

$$
\pi_{t}^{*}=\frac{1}{1-\eta} \cdot \frac{\mu-r}{\left(k S_{t}^{\gamma}\right)^{2}} X_{t}\left(1-\frac{2 \gamma(1-\eta) I(t)}{\mu-r}\right),
$$

where $I(t)$ is given by (33). This is just the optimal policy for power utility maximizing when consumption is not considered and stock price follows a CEV process.

Special Case 3. If $\eta \rightarrow 0$, then $B(t)=0$ and $A(t)=\beta(t-T)$. In addition, we have

$$
h(t, y)=\frac{\alpha}{\beta}\left(1-e^{-\beta(T-t)}\right)+(1-\alpha) e^{-\beta(T-t)}
$$

and $(\partial h / \partial y) / h=0$. Therefore, $(36)$ is reduced to

$$
\begin{gathered}
\pi_{t}^{*}=\frac{\mu-r}{\left(k S_{t}^{\gamma}\right)^{2}} X_{t}, \\
C_{t}^{*}=\frac{\alpha}{(\alpha / \beta)\left(1-e^{-\beta(T-t)}\right)+(1-\alpha) e^{-\beta(T-t)}} X_{t} .
\end{gathered}
$$

It is well known that power utility is degenerated to logarithm utility if $\eta \rightarrow 0$. Furthermore, we find that the optimal investment and consumption strategy obtained by (43) is just that under logarithm utility function.

3.2. Logarithm Utility. Logarithm utility function is defined as

$$
U_{1}(x)=U_{2}(x)=\ln x
$$

We can assume that the solution to the HJB equation (9) is of the following structure:

$$
\begin{aligned}
& H(t, s, x)=v(t) e^{-\beta t} \ln x+w(t, s), \\
& v(T)=1-\alpha, \quad w(T, s)=0 .
\end{aligned}
$$

Then, partial derivatives with respect to $t, s$, and $x$ are given by

$$
\begin{aligned}
& \frac{\partial H}{\partial t}=v^{\prime}(t) e^{-\beta t} \ln x-\beta v(t) e^{-\beta t} \ln x+\frac{\partial w}{\partial t}, \\
& \frac{\partial H}{\partial x}=v(t) e^{-\beta t} \frac{1}{x}, \quad \frac{\partial^{2} H}{\partial x^{2}}=v(t) e^{-\beta t} \frac{-1}{x^{2}}, \\
& \frac{\partial H}{\partial s}=\frac{\partial w}{\partial s}, \quad \frac{\partial^{2} H}{\partial s^{2}}=\frac{\partial^{2} w}{\partial s^{2}}, \quad \frac{\partial^{2} H}{\partial s \partial x}=0 .
\end{aligned}
$$

Substituting these partial derivatives back into (9) yields

$$
\begin{aligned}
\frac{\partial w}{\partial t} & +\mu s \frac{\partial w}{\partial s}+\frac{1}{2} k^{2} s^{2 \gamma+2} \frac{\partial^{2} w}{\partial s^{2}}+\left(r+\frac{1}{2}\left(\frac{\mu-r}{k s^{\gamma}}\right)^{2}\right) e^{-\beta t} v(t) \\
& +\alpha e^{-\beta t}(\ln \alpha-\ln v(t)-1) \\
& +e^{-\beta t} \ln x\left(\frac{d v(t)}{d t}-\beta v(t)+\alpha\right)=0
\end{aligned}
$$


We can split this equation into the following two equations:

$$
\begin{gathered}
\frac{d v(t)}{d t}-\beta v(t)+\alpha=0, \quad v(T)=1-\alpha \\
\frac{\partial w}{\partial t}+\mu s \frac{\partial w}{\partial s}+\frac{1}{2} k^{2} s^{2 \gamma+2} \frac{\partial^{2} w}{\partial s^{2}}+\left(r+\frac{1}{2}\left(\frac{\mu-r}{k s^{\gamma}}\right)^{2}\right) e^{-\beta t} v(t) \\
+\alpha e^{-\beta t}(\ln \alpha-\ln v(t)-1)=0, \quad w(T, s)=0
\end{gathered}
$$

Solving (48), we get

$$
v(t)=(1-\alpha) e^{-\beta(T-t)}-\frac{\alpha}{\beta}\left(e^{-\beta(T-t)}-1\right) .
$$

Lemma 8. For (49), one tries to fit a solution of the form $w(t, s)=D(t)+E(t) y, y=s^{-2 \gamma}$, with boundary conditions $D(T)=0$ and $E(T)=0$; then $E(t)$ and $D(t)$ are given by (55) and (56), respectively.

Proof. Note that

$$
\begin{aligned}
& \frac{\partial w}{\partial t}=\frac{d D(t)}{d t}+\frac{d E(t)}{d t} y, \quad \frac{\partial w}{\partial s}=E(t)(-2 \gamma) s^{-2 \gamma-1}, \\
& \frac{\partial^{2} w}{\partial s^{2}}=E(t)(-2 \gamma)(-2 \gamma-1) s^{-2 \gamma-2} .
\end{aligned}
$$

Introducing these derivatives into (49), we get

$$
\begin{gathered}
y\left[\frac{d E(t)}{d t}-2 \gamma \mu E(t)+\frac{1}{2}\left(\frac{\mu-r}{k}\right)^{2} e^{-\beta t} v(t)\right] \\
+\frac{d D(t)}{d t}+r e^{-\beta t} v(t)+\gamma(2 \gamma+1) k^{2} E(t) \\
+\alpha e^{-\beta t}(\ln \alpha-\ln v(t)-1)=0 .
\end{gathered}
$$

Then we can decompose this equation into two equations:

$$
\begin{aligned}
& \frac{d E(t)}{d t}-2 \gamma \mu E(t)+\frac{1}{2}\left(\frac{\mu-r}{k}\right)^{2} e^{-\beta t} v(t)=0, \quad E(T)=0 \\
& \frac{d D(t)}{d t}+r e^{-\beta t} v(t)+\gamma(2 \gamma+1) k^{2} E(t) \\
& +\alpha e^{-\beta t}(\ln \alpha-\ln v(t)-1)=0, \quad D(T)=0
\end{aligned}
$$

Solving (53) and (54) yields

$$
\begin{aligned}
E(t)= & \frac{1}{2}\left(\frac{\mu-r}{k}\right)^{2} \frac{\beta-\alpha \beta-\alpha}{2 \beta \gamma \mu} e^{-\beta T}\left(1-e^{-2 \gamma \mu(T-t)}\right) \\
& +\frac{1}{2}\left(\frac{\mu-r}{k}\right)^{2} \frac{\alpha}{\beta(\beta+2 \gamma \mu)} e^{-\beta T}\left(e^{\beta(T-t)}-e^{-2 \gamma \mu(T-t)}\right), \\
D(t)= & \int_{t}^{T} r e^{-\beta t} v(t) d t+\gamma(2 \gamma+1) k^{2} \int_{t}^{T} E(t) d t-\alpha \\
& \times \int_{t}^{T} e^{-\beta t} \ln v(t) d t-\frac{\alpha(\ln \alpha-1)}{\beta}\left(e^{-\beta T}-e^{-\beta t}\right) .
\end{aligned}
$$

At the end, Lemma 8 is proved.
Noting that

$$
\frac{\partial H / \partial x}{\partial^{2} H / \partial x^{2}}=-x, \quad \frac{\partial^{2} H / \partial x \partial s}{\partial^{2} H / \partial x^{2}}=0,
$$

we can rewrite the optimal investment and consumption strategy as

$$
\pi_{t}^{*}=\frac{\mu-r}{\left(k s^{\gamma}\right)^{2}} x, \quad C_{t}^{*}=\frac{\alpha}{v(t)} x .
$$

Finally, we can conclude the optimal investment and consumption strategy for logarithm utility in the following theorem.

Theorem 9. If utility function is given by $U_{1}(x)=U_{2}(x)=$ $\ln x$, the optimal investment and consumption policy of problem (5) is

$$
\pi_{t}^{*}=\frac{\mu-r}{\left(k S_{t}^{\gamma}\right)^{2}} X_{t}
$$

$$
C_{t}^{*}=\frac{\alpha}{(1-\alpha) e^{-\beta(T-t)}-(\alpha / \beta)\left(e^{-\beta(T-t)}-1\right)} X_{t}
$$

Remark 10. It can be seen from (59) that the optimal investment strategy $\pi_{t}^{*}$ is not correlated with the parameters $\alpha$ and $\beta$. And when $\alpha \rightarrow 0$, the optimal consumption policy is $C_{t}^{*}=0$ and the optimal portfolios are still given by $\pi_{t}^{*}=$ $\left((\mu-r) /\left(k S_{t}^{\gamma}\right)^{2}\right) X_{t}$.

\section{Numerical Analysis}

This section provides a numerical example to illustrate the dynamic behavior of the optimal investment and consumption strategy in the power utility case. Assume that there are two assets in the financial market; one is a bond and the other is a stock. Throughout the numerical analysis, unless otherwise stated, the basic parameters are given by $r=0.03$, $\mu=0.12, k=16.16, \gamma=-1, S_{0}=67, t=0, T=2, x_{0}=100$, and $\eta=-2$. Notice that the first five parameters are taken from the paper of Gao [15]; without loss of a generality, we consider the initial time $t=0$ and the initial wealth $x_{0}=100$.

4.1. Sensitivity Analysis for the Optimal Investment Strategy. Figures 1, 2, and 3 provide some sensitivity analysis of market parameters on the optimal investment strategy. Some results and economic implications can be summarized as follows.

(a1) The optimal investment strategy $\pi_{t}^{*}$ decreases with respect to interest rate $r$. When risk-free interest rate is increasing, the income from investment in the riskfree asset will be more and more bigger; accordingly, the investor will reduce the investment amount in the stock in order to avoid the risk form investments. It is consistent with the intuition.

(a2) $\pi_{t}^{*}$ is increasing as the parameter $\mu$ becomes bigger. In fact, as the value of $\mu$ is increasing, the appreciate rate of the stock is increasing; the investor would invest more money in the stock in order to gain more revenue. 

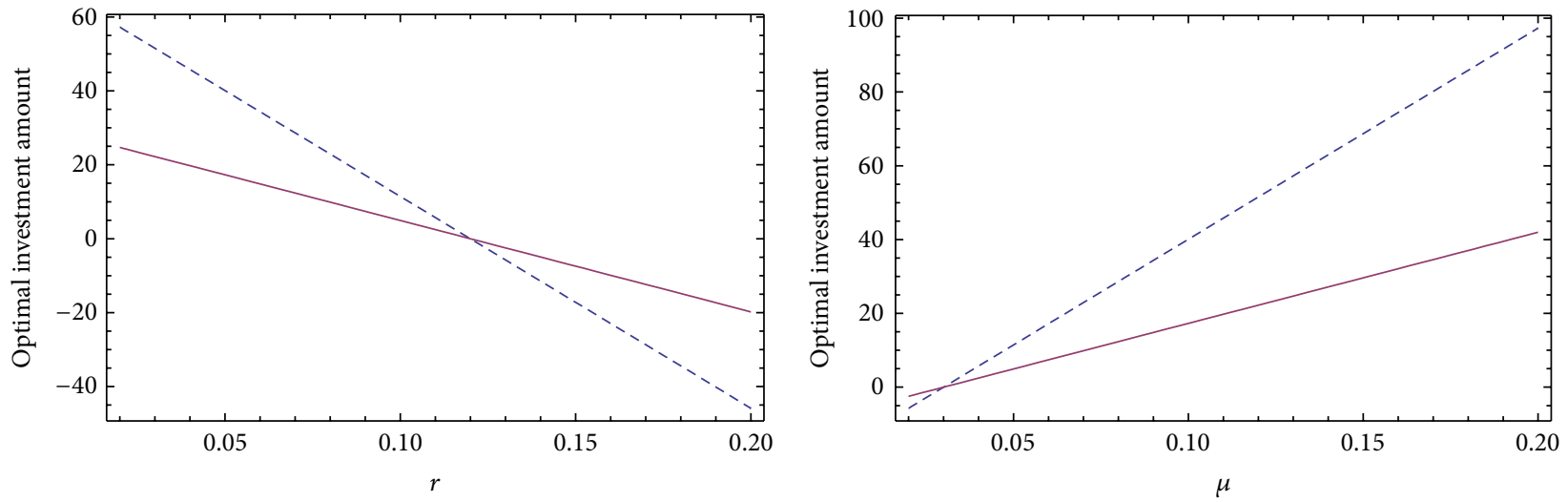

$\begin{aligned}--\gamma & =-1 \\ -\gamma & =-0.9\end{aligned}$

- - $\gamma=-1$

$-\gamma=-0.9$

(a)

(b)

FIgURE 1: The impact of $r$ and $\mu$ on $\pi_{t}^{*}$.

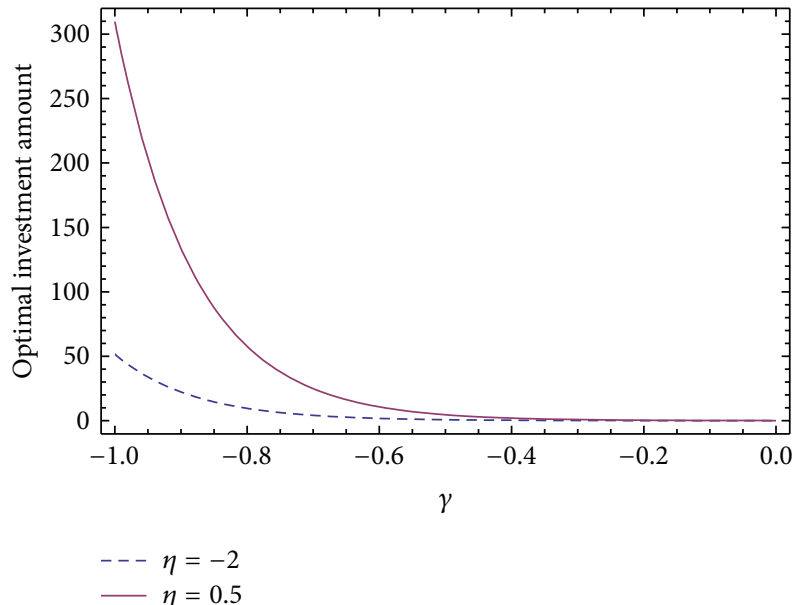

(a)

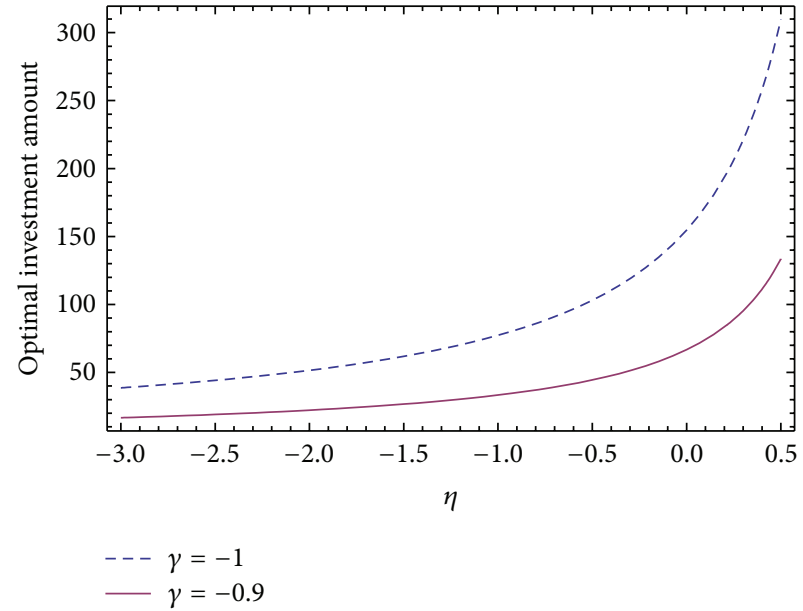

(b)

FIGURE 2: The impact of $\gamma$ and $\eta$ on $\pi_{t}^{*}$.

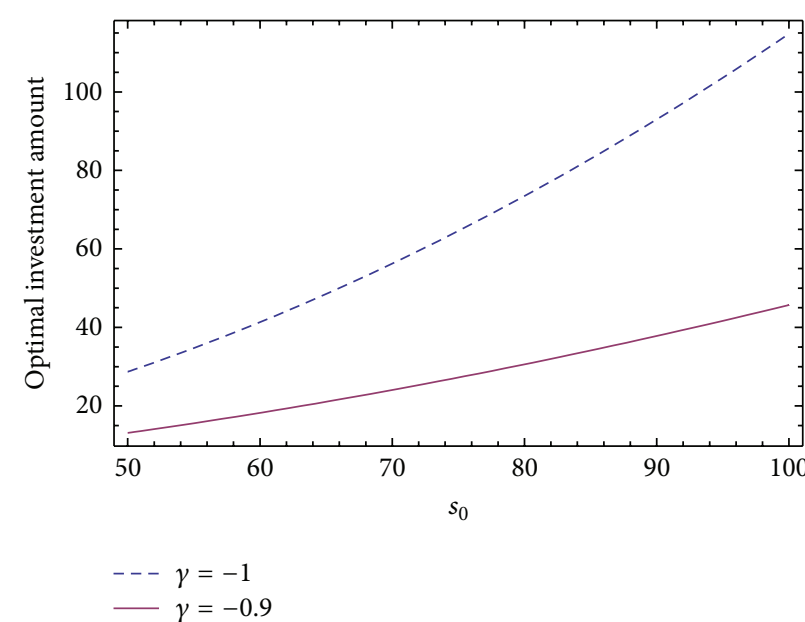

(a)

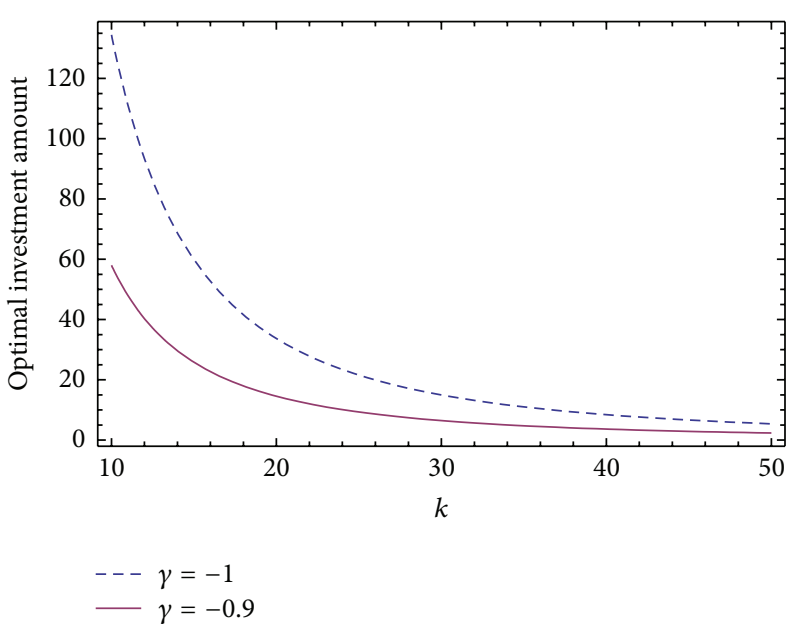

(b)

FIgURE 3: The impact of $s_{0}$ and $k$ on $\pi_{t}^{*}$. 


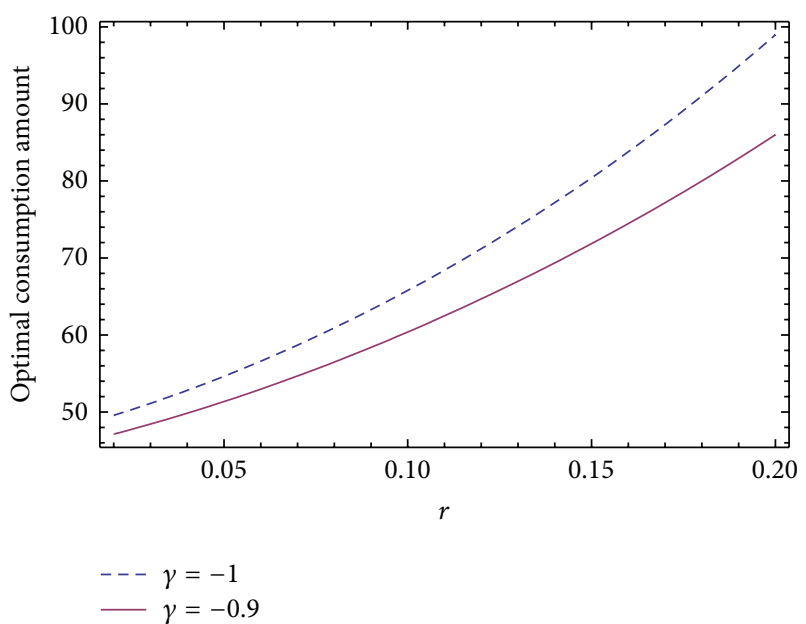

(a)

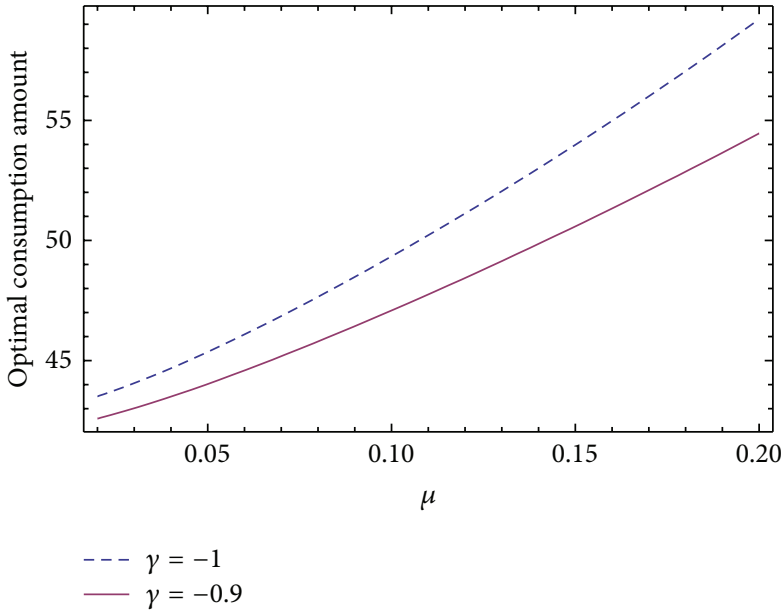

(b)

Figure 4: The impact of $r$ and $\mu$ on $C_{t}^{*}$.

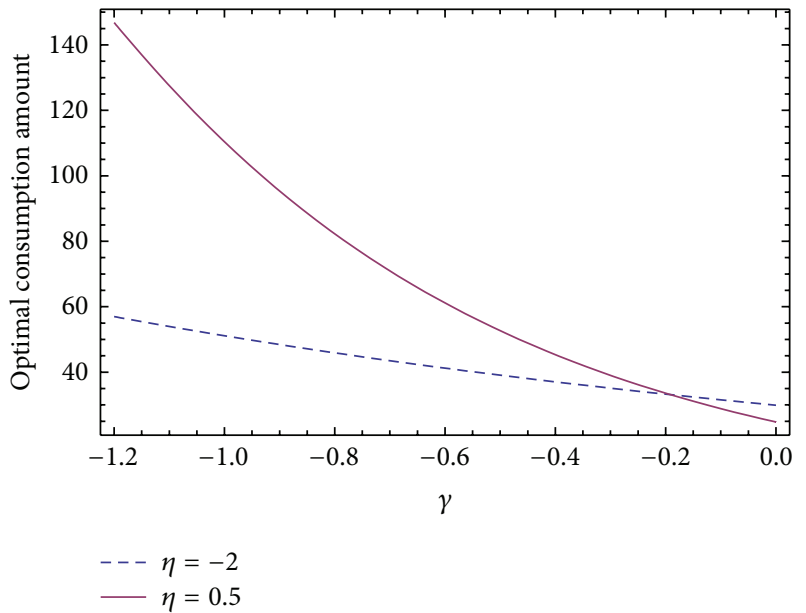

(a)

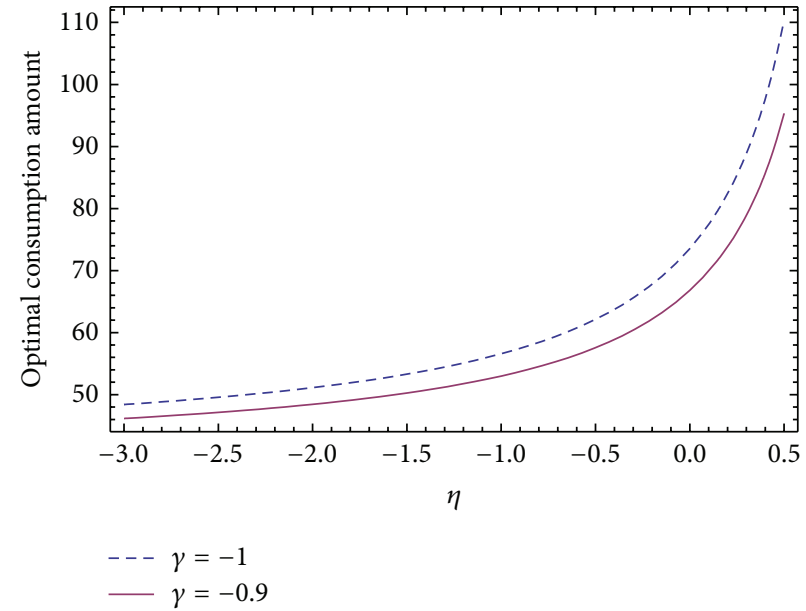

(b)

Figure 5: The impact of $\gamma$ and $\eta$ on $C_{t}^{*}$.
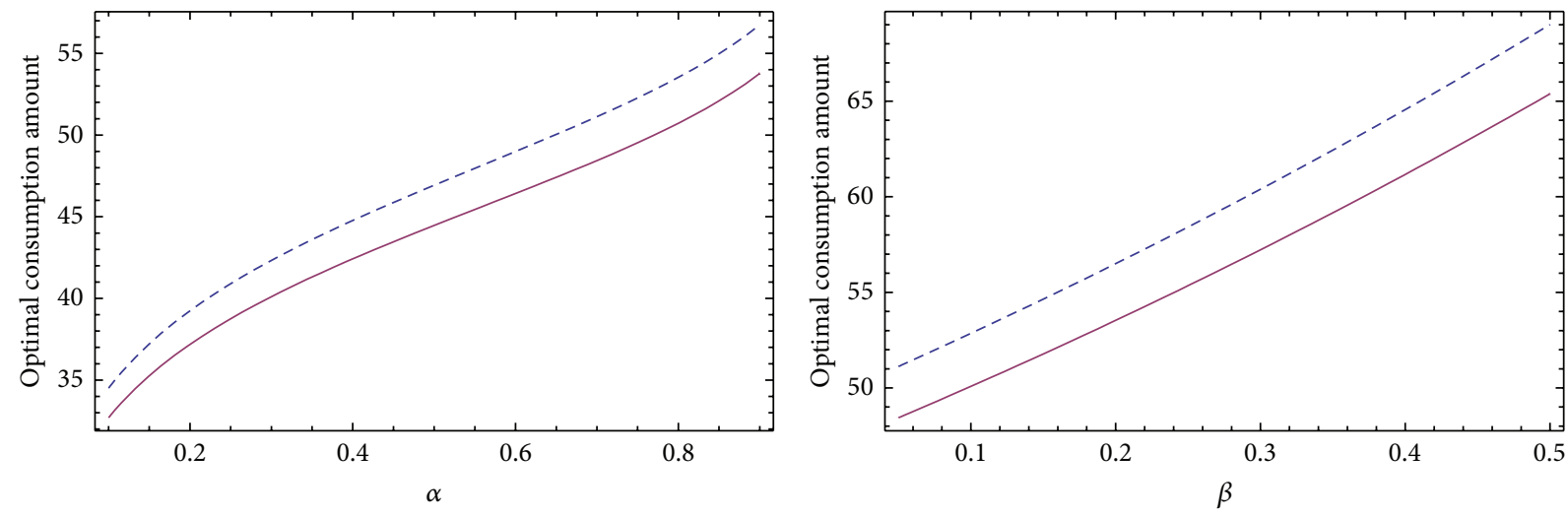

$\begin{aligned}---\gamma & =-1 \\ -\gamma & =-0.9\end{aligned}$

$\begin{aligned}--\gamma & =-1 \\ -\gamma & =-0.9\end{aligned}$

(a)

(b)

Figure 6: The impact of $\alpha$ and $\beta$ on $C_{t}^{*}$. 
(a3) $\pi_{t}^{*}$ is decreasing in $\gamma$. As a matter of fact, when elasticity parameter $\gamma$ (under the constraints $\gamma<$ 0 and $r<\mu$ ) becomes larger, the instantaneous volatility $k S_{t}^{\gamma}$ of the stock will be more and more bigger, which leads to the more risk of investment. Therefore, the investor needs to reduce the amount invested in the stock. Moreover, we can draw the conclusion that the amount invested in the stock under a CEV model is more than that under a GBM model.

(a4) $\pi_{t}^{*}$ is increasing with the aversion factor of risk $\eta$ increasing. In reality, the aversion coefficient of risk is given by $1-\eta$ in the power utility case. It means that the bigger the value of $\eta$ is, the less the risk aversion of the investor is. It leads to that the investor would invest more money in the stock in order to get more income.

(a5) $\pi_{t}^{*}$ increases with respect to stock price $s_{0}$. It is completely different from the optimal investment strategy under a geometric Brownian motion. From Special Case 1, we can get that the optimal policy under a GBM is not correlated with stock price. In fact, under the CEV model, the instantaneous volatility $k S_{t}^{\gamma}$ of the stock decreases when stock price is increasing. So the risk of investments is decreasing; accordingly, the investor has more willing to invest more money in the stock in order to get more wealth.

(a6) $\pi_{t}^{*}$ has opposite trend with the value of $k$. Actually, the larger the value of $k$ is, the bigger the instantaneous volatility $k S_{t}^{\gamma}$ of the stock is, which leads to the more risk of investment. Therefore, the investor should invest less money in the stock.

4.2. Sensitivity Analysis for the Optimal Consumption Strategy. From Figures 4, 5, and 6, we can draw the following conclusions.

(b1) The optimal consumption strategy $C_{t}^{*}$ is increasing in interest rate $r$. In fact, the bigger the value of risk-free interest rate $r$ is, the more the amount invested in the risk-free asset is, and meantime the less the amount in the stock is. But the total expected wealth of the investor will become more and more larger. Therefore, The amount the investor can consume will increase accordingly.

(b2) $C_{t}^{*}$ has same trend with the appreciation rate of the stock $\mu$. It implies that when the value of $\mu$ is increasing, the amount invested in the stock is rising, which leads to that the expected wealth of an investor will increase as well. Therefore, the optimal consumption amount will become more and more bigger.

(b3) $C_{t}^{*}$ is decreasing in $\gamma$. This displays that the bigger the value of elasticity parameter $\gamma$ is, the less the optimal consumption amount is. As a matter of fact, when the value of $\gamma$ becomes larger, the amount invested in the stock is decreasing, which leads to that the expected wealth of an investor will become less. So the amount to consume will decrease.

(b4) $C_{t}^{*}$ increases with respect to the risk aversion factor $\eta$. According to the above analysis, when the value of $\eta$ is increasing, the amount invested in the stock is also increasing, which results in that the expected wealth of an investor is rising meantime. Therefore, an investor can consume more money.

(b5) $C_{t}^{*}$ is increasing as the value of $\alpha$ is rising. When the parameter $\alpha$ becomes larger, the relative importance of consumption will become bigger between consumption and terminal wealth. Therefore, an investor must add the amount to consume.

(b6) $C_{t}^{*}$ has the same trend with discount factor $\beta$. It implies that the larger the value of $\beta$ is, the more the amount to consume is.

\section{Conclusions}

In this paper, we have investigated an investment and consumption problem, in which stock price is assumed to follow a CEV process. The CEV process is a natural extension of a geometric Brownian motion. It is one of the most important innovations. By applying dynamic programming principle and variable change technique, we derive the closed-form solutions to the optimal investment and consumption strategies in the power and logarithm utility cases. Finally, we propose a numerical example to illustrate the impact of market parameters on the optimal investment and consumption strategies and give some economic implications. Some important conclusions are found: (i) $\pi_{t}^{*}$ and $C_{t}^{*}$ have opposite trend in risk-free interest rate $r$; (ii) $\pi_{t}^{*}$ and $C_{t}^{*}$ have same trend in the parameters $\mu, \gamma$, and $\eta$.

In future research on the investment and consumption problem, we focus on the cases of more sophisticated situations such as introducing transaction costs, stochastic affine interest rate, and the other uncertain factors, which would result in more sophisticated nonlinear second-order partial differential equations and cannot deal with them. We leave these points to future research.

\section{Acknowledgments}

This research is supported by National Natural Science Foundation of China (no. 11301376), Humanities and Social Science Research Youth Foundation of Ministry of Education of China (no. 11YJC790006), and Higher School Science and Technology Development Foundation of Tianjin (no. 20100821).

\section{References}

[1] R. C. Merton, "Lifetime portfolio selection under uncertainty: the continuous-time case," The Review of Economics and Statistics, vol. 51, no. 3, pp. 247-257, 1969.

[2] R. C. Merton, "Optimum consumption and portfolio rules in a continuous-time model," Journal of Economic Theory, vol. 3, no. 4, pp. 373-413, 1971. 
[3] S. E. Shreve and H. M. Soner, "Optimal investment and consumption with transaction costs," The Annals of Applied Probability, vol. 4, no. 3, pp. 609-692, 1994.

[4] M. Akian, J. L. Menaldi, and A. Sulem, "On an investmentconsumption model with transaction costs," SIAM Journal on Control and Optimization, vol. 34, no. 1, pp. 329-364, 1996.

[5] K. Janeček and S. E. Shreve, "Asymptotic analysis for optimal investment and consumption with transaction costs," Finance and Stochastics, vol. 8, no. 2, pp. 181-206, 2004.

[6] W. H. Fleming and T. Zariphopoulou, "An optimal investment/consumption model with borrowing," Mathematics of Operations Research, vol. 16, no. 4, pp. 802-822, 1991.

[7] J.-L. Vila and T. Zariphopoulou, "Optimal consumption and portfolio choice with borrowing constraints," Journal of Economic Theory, vol. 77, no. 2, pp. 402-431, 1997.

[8] R. Yao and H. H. Zhang, "Optimal consumption and portfolio choices with risky housing and borrowing constraints," The Review of Financial Studies, vol. 18, no. 1, pp. 197-239, 2005.

[9] J. C. Cox and S. A. Ross, "The valuation of options for alternative stochastic processes," Journal of Financial Economics, vol. 3, no. 1-2, pp. 145-166, 1976.

[10] M. Schroder, "Computing the constant elasticity of variance option pricing formula," Journal of Finance, vol. 44, no. 1, pp. 211-219, 1989.

[11] C. F. Lo, P. H. Yuen, and C. H. Hui, "Constant elasticity of variance option pricing model with time-dependent parameters," International Journal of Theoretical and Applied Finance, vol. 3, no. 4, pp. 661-674, 2000.

[12] P. B. Phelim and S. T. Yisong, "Pricing lookback and barrier options under the CEV process," Journal of Financial and Quantitative Analysis, vol. 34, no. 2, pp. 241-264, 1999.

[13] D. Davydov and V. Linetsky, "Pricing and hedging pathdependent options under the CEV process," Management Science, vol. 47, no. 7, pp. 949-965, 2001.

[14] J. Xiao, Z. Hong, and C. Qin, "The constant elasticity of variance (CEV) model and the Legendre transform-dual solution for annuity contracts," Insurance: Mathematics and Economics, vol. 40, no. 2, pp. 302-310, 2007.

[15] J. Gao, "Optimal investment strategy for annuity contracts under the constant elasticity of variance (CEV) model," Insurance: Mathematics and Economics, vol. 45, no. 1, pp. 9-18, 2009.

[16] J. Gao, "An extended CEV model and the Legendre transformdual-asymptotic solutions for annuity contracts," Insurance: Mathematics and Economics, vol. 46, no. 3, pp. 511-530, 2010.

[17] M. Gu, Y. Yang, S. Li, and J. Zhang, "Constant elasticity of variance model for proportional reinsurance and investment strategies," Insurance: Mathematics and Economics, vol. 46, no. 3, pp. 580-587, 2010

[18] X. Lin and Y. Li, "Optimal reinsurance and investment for a jump diffusion risk process under the CEV model," North American Actuarial Journal, vol. 15, no. 3, pp. 417-431, 2011.

[19] A. Gu, X. Guo, Z. Li, and Y. Zeng, "Optimal control of excessof-loss reinsurance and investment for insurers under a CEV model," Insurance: Mathematics and Economics, vol. 51, no. 3, pp. 674-684, 2012.

[20] E. J. Jung and J. H. Kim, "Optimal investment strategies for the HARA utility under the constant elasticity of variance model," Insurance: Mathematics and Economics, vol. 51, no. 1, pp. 667673, 2012.

[21] H. Zhao and X. Rong, "Portfolio selection problem with multiple risky assets under the constant elasticity of variance model," Insurance: Mathematics and Economics, vol. 50, no. 1, pp. 179-190, 2012.

[22] J. Liu, "Portfolio selection in stochastic environments," The Review of Financial Studies, vol. 20, no. 1, pp. 1-39, 2007. 


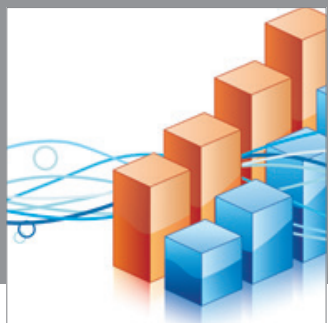

Advances in

Operations Research

mansans

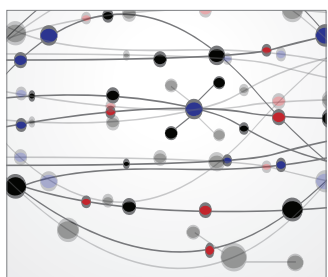

The Scientific World Journal
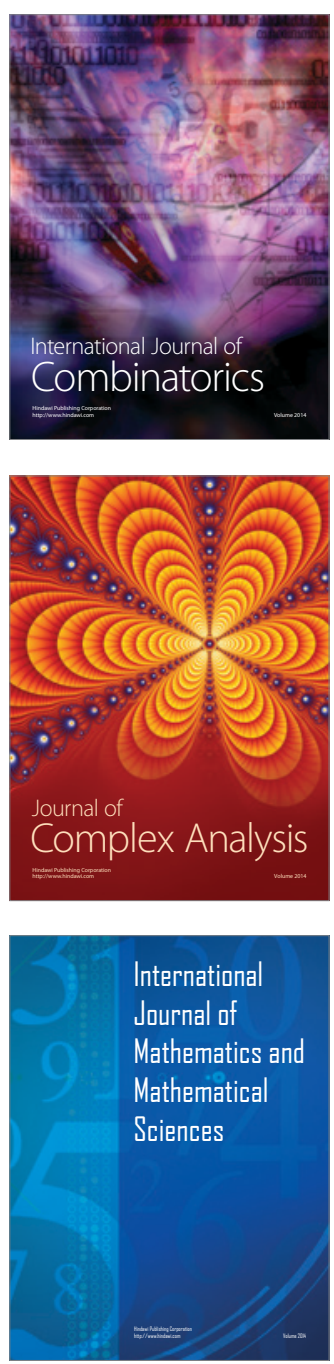
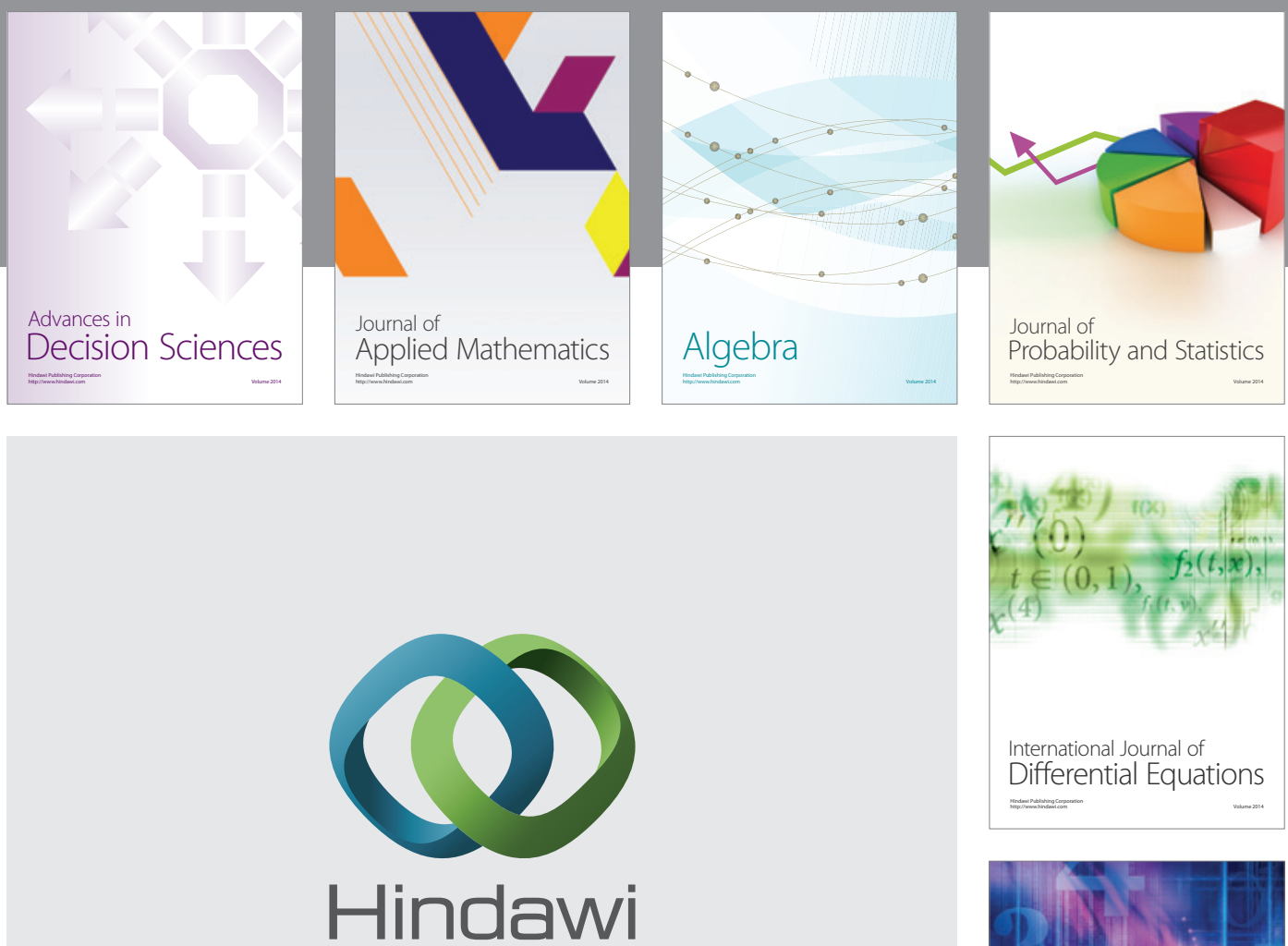

Submit your manuscripts at http://www.hindawi.com
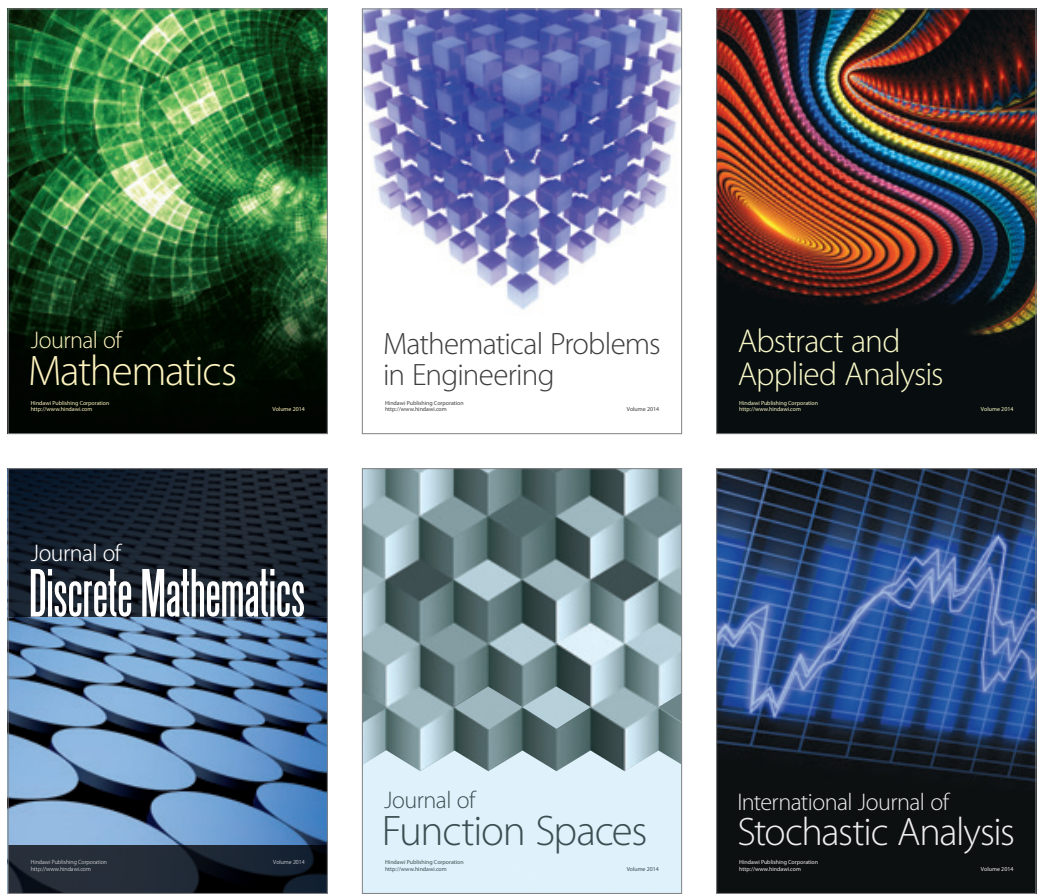

Journal of

Function Spaces

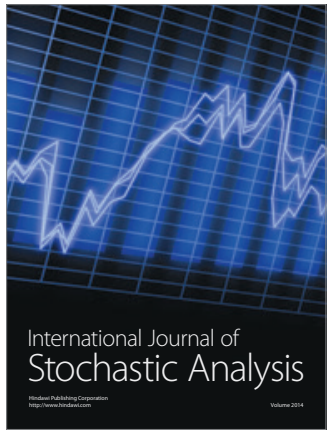

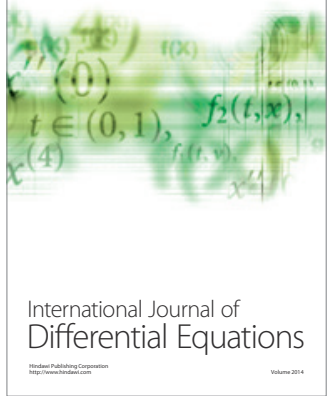
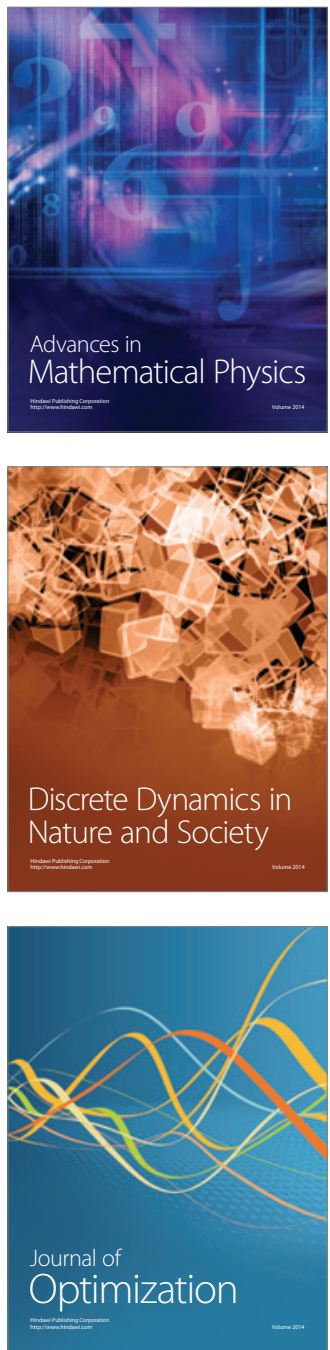